\title{
Total Selective Synthesis of Enantiopure $O^{1}$-Acyl-3-
}

\section{aminoalkan-1,2-diols by Ring-Opening of Amino Epoxides \\ with Carboxylic Acids.}

\author{
José M. Concellón,* José Ramón Suárez, Virginia del Solar, and Ricardo Llavona \\ Departamento de Química Orgánica e Inorgánica, Facultad \\ de Química, Universidad de Oviedo, Julián Clavería, 8, \\ 33071 Oviedo, Spain. \\ Email:jmcg@fq.uniovi.es
}

General: All reactions were carried out under an atmosphere of dry $\mathrm{N}_{2}$ using oven-dried glassware and syringes. All reagents were purchased in the higher quality available and were used without further purification. $\mathrm{BF}_{3} \mathrm{OEt}_{2}$ was distilled from $\mathrm{CaH}_{2}$ and stored over activated $4 \AA$ molecular sieves. The solvents used in column chromatography, were obtained from commercial suppliers and used without further distillation. TLC was performed on aluminium-backed plates coated with silica gel 60 with $\mathrm{F}_{254}$ indicator (Scharlau). Flash column chromatography was carried out on silica gel 60, 230-240 mesh. ${ }^{1} \mathrm{H}$ NMR (200, 300, $400 \mathrm{MHz})$ and ${ }^{13} \mathrm{C} \operatorname{NMR}(50,75,100 \mathrm{MHz})$ spectra were measured at room temperature, with tetramethylsilane $\left(\delta=0.0,{ }^{1} \mathrm{H} \mathrm{NMR}\right)$ or $\mathrm{CDCl}_{3}\left(\delta=77.00,{ }^{13} \mathrm{C} \mathrm{NMR}\right)$ as internal standard. Carbon multiplicities were assigned by DEPT techniques. Low-resolution electron impact mass spectra (EI-LRMS) were obtained at $70 \mathrm{eV}$, and the intensities are reported as a percentage relative to the base peak after the corresponding $\mathrm{m} / \mathrm{z}$ value.

\footnotetext{
${ }^{13} \mathrm{C}-\mathrm{RMN}$ of 5,8 and $9:$

(2R,3S)-O,O-Diacetyl-3-dibenzylamino-5-methylhexan-1,2-diol (5a). $3 \mathrm{~S}$

(2R,3S)-O,O-Diacetyl-3-dibenzylamino-4-phenylbutan-1,2-diol (5b) $3 \mathrm{~S}$

(2R,3S)-O,O-Dicinamoyl-3-dibenzylamino-4-phenylbutan-1,2-diol (5c)..... $4 \mathrm{~S}$

$(2 R, 3 S)-O^{I}$-Hexanoyl-3-dibenzylaminobutan-1,2-diol (8a). $4 \mathrm{~S}$

(2R,3S)- $O^{I}$-(3-Phenylpropenoyl)-3-dibenzylaminobutan-1,2-diol (8b). $5 \mathrm{~S}$

$(2 R, 3 S)-O^{I}$-Acetyl-3-dibenzylamino-5-methylhexan-1,2-diol (8c) $5 \mathrm{~S}$
} 
(2R,3S)- $O^{I}$-Hexanoyl-3-dibenzylamino-5-methylhexan-1,2-diol (8d).

$(2 R, 3 S)-O^{I}$-Acetyl-3-dibenzylamino-4-phenylbutan-1,2-diol (8e)... $6 \mathrm{~S}$

(2R,3S)- $O^{I}$-Penta-2-enoyl-3-dibenzylamino-4-phenylbutan-1,2-diol (8f)... $7 \mathrm{~S}$

(2S,3S)- $O^{I}$-Penta-2-enoyl-3-dibenzylaminobutan-1,2-diol (9a). $7 \mathrm{~S}$

(2S,3S)- $O^{I}$-Hexanoyl-3-dibenzylaminobutan-1,2-diol (9b). $8 \mathrm{~S}$

(2S,3S)- $O^{I}$-Acetyl-3-dibenzylamino-5-methylhexan-1,2-diol (9c). $8 \mathrm{~S}$

(2S,3S)- $O^{I}$-Hexanoyl-3-dibenzylamino-5-methylhexan-1,2-diol (9d) 9S

(2S,3S)- $O^{I}$-Acetyl-3-dibenzylamino-4-phenylbutan-1,2-diol (9e)...... 9S

(2S,3S)- $O^{I}$-Penta-2-enoyl-3-dibenzylamino-4-phenylbutan-1,2-diol (9f). $10 \mathrm{~S}$ 


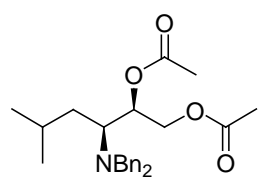

$5 a$
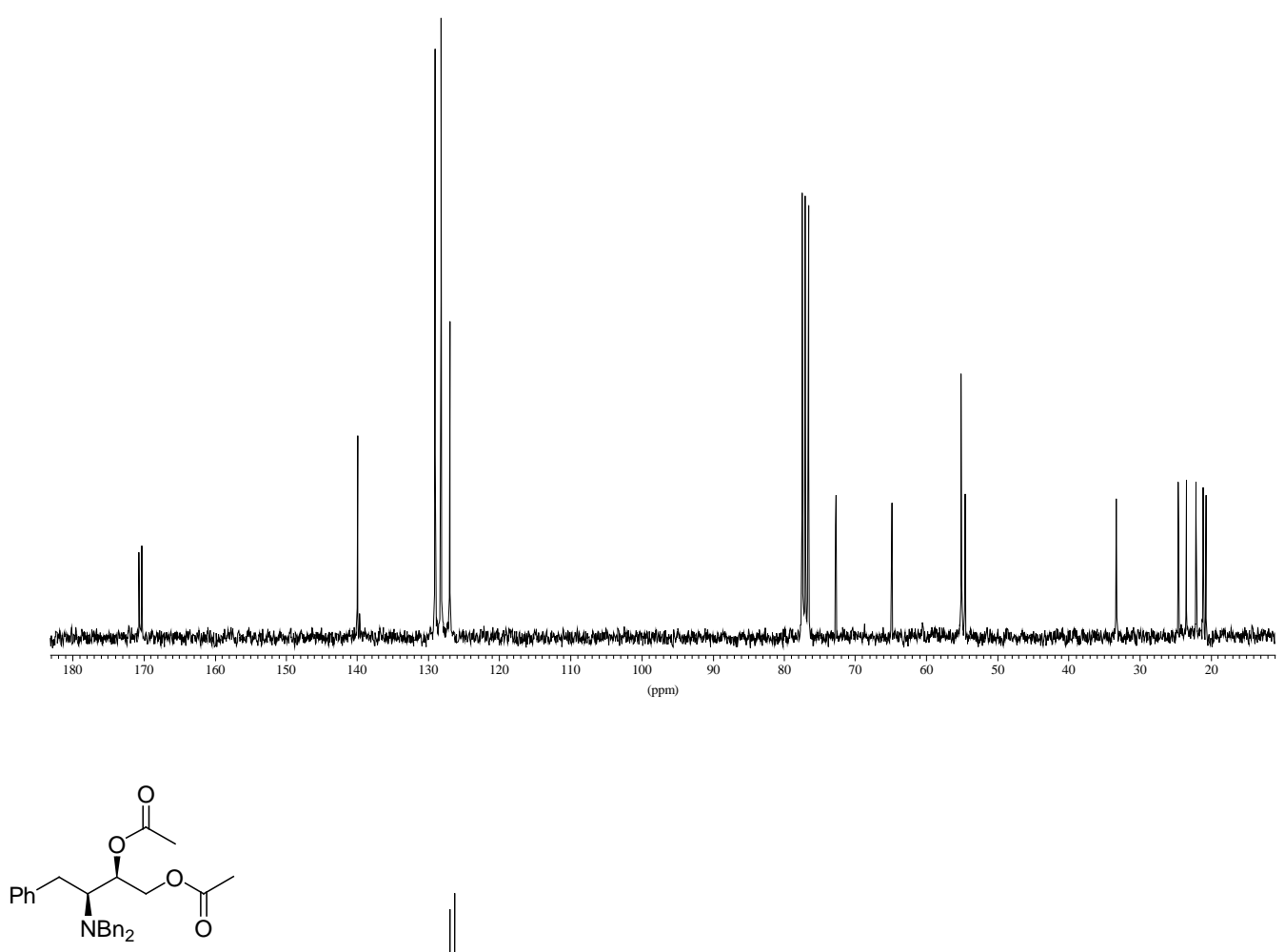

5b

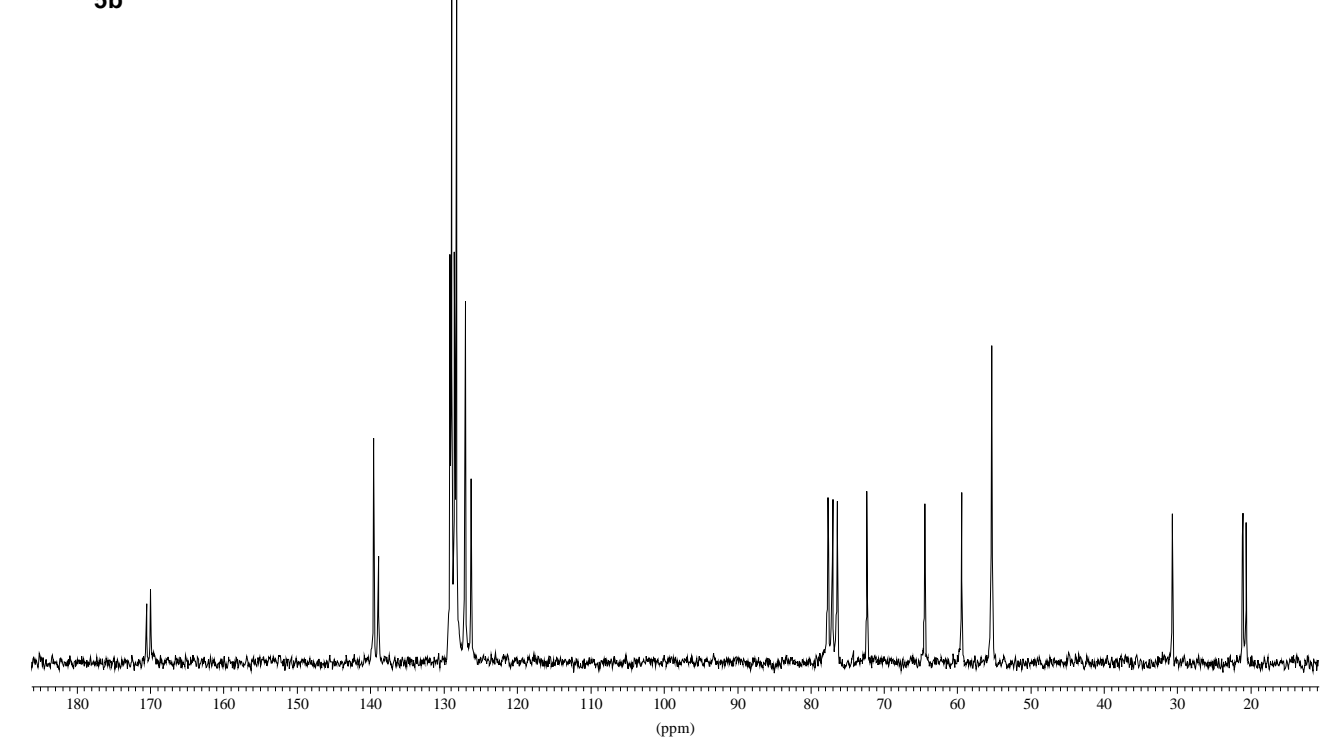



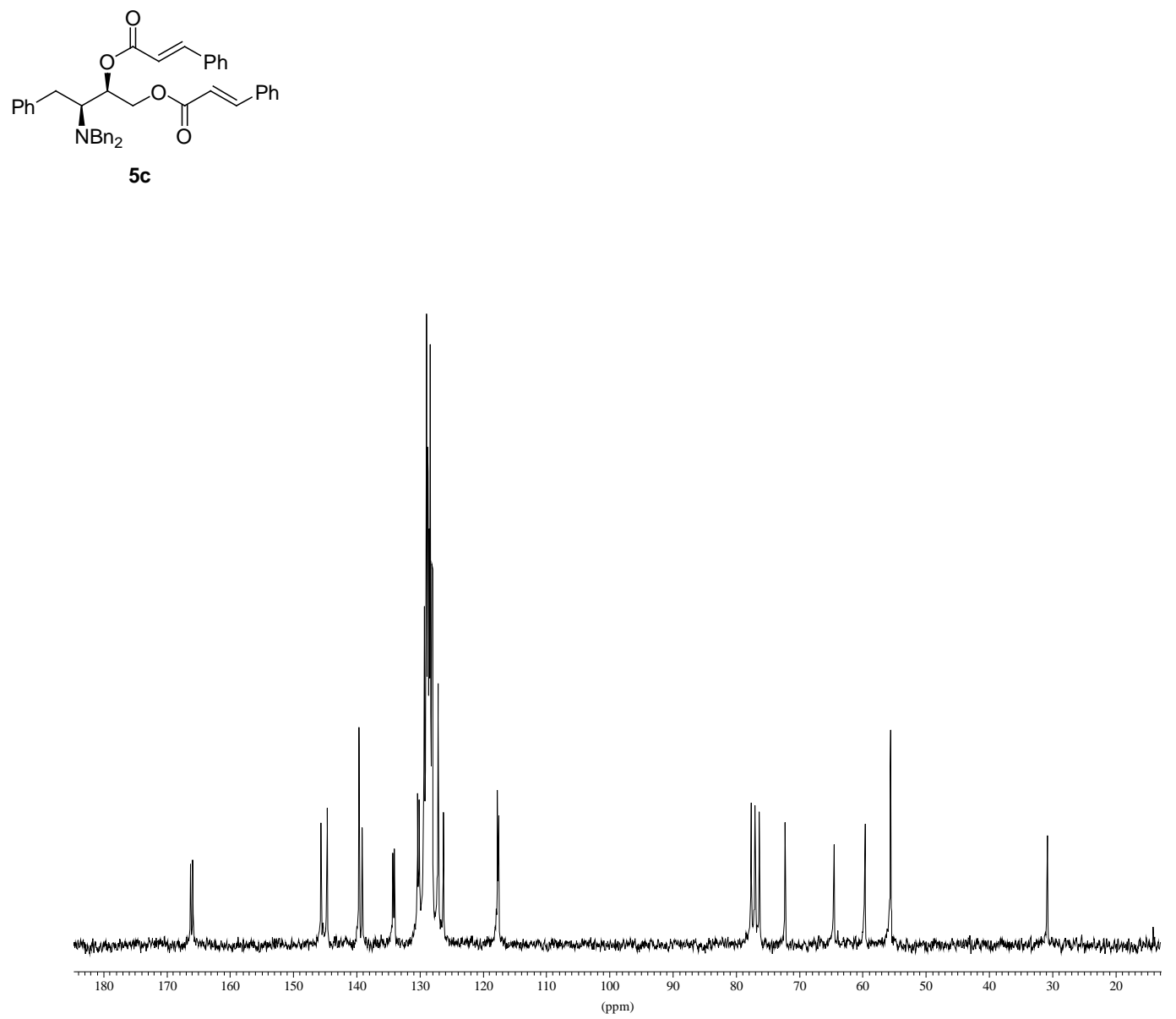<smiles>CCCCCC(=O)OCC(O)C(C)[N+](=O)[O-]</smiles>

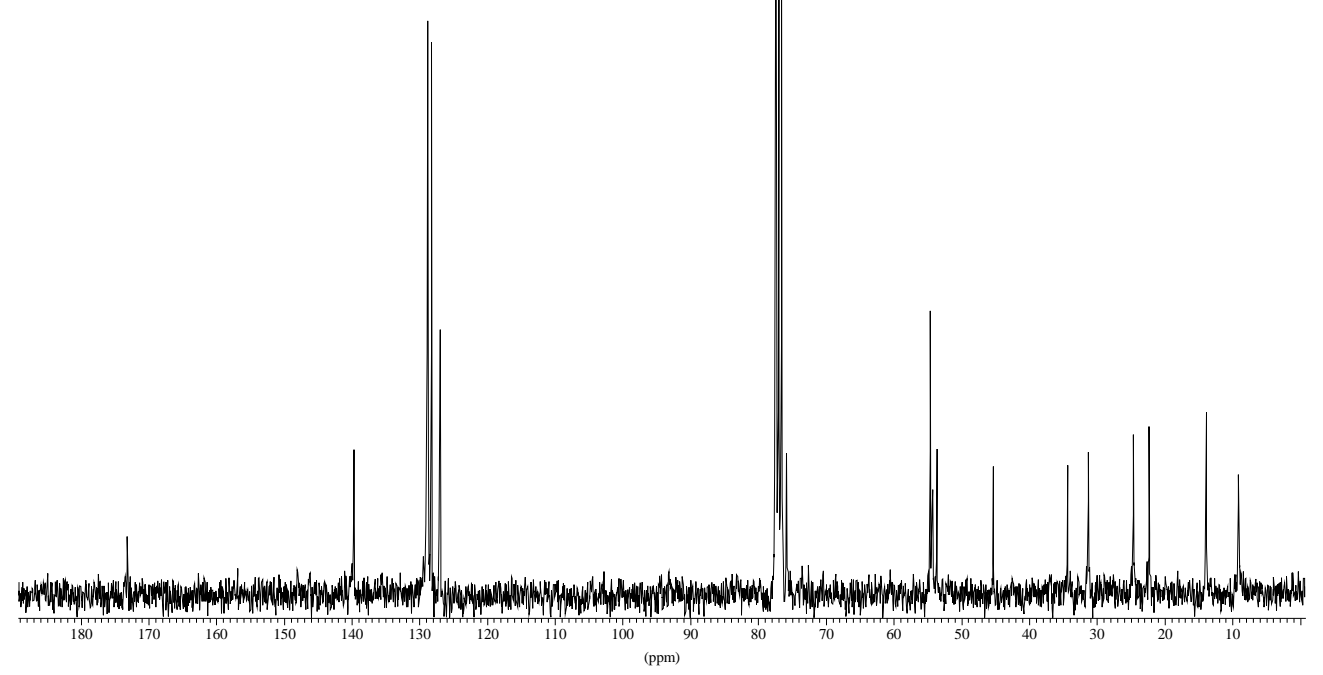



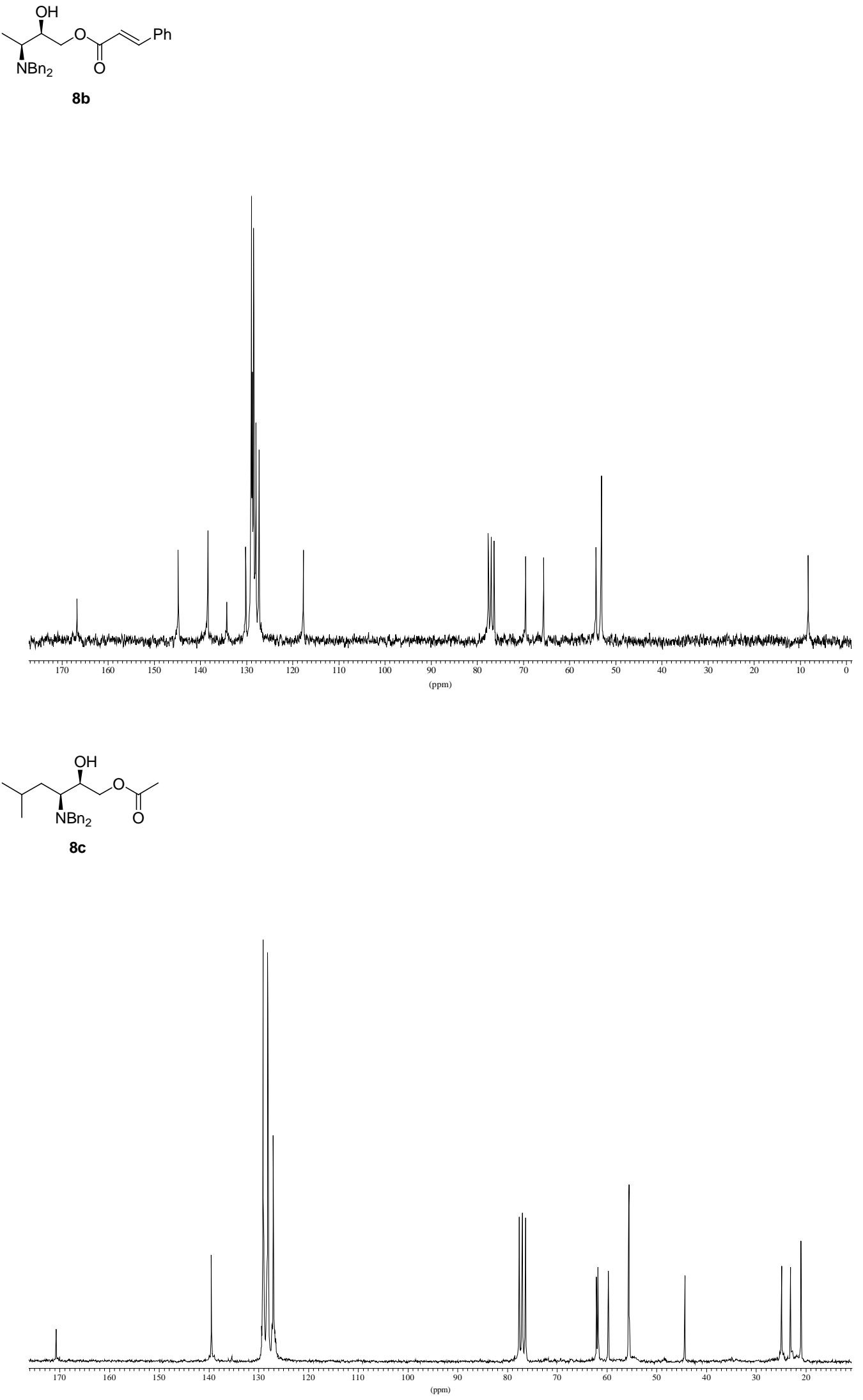

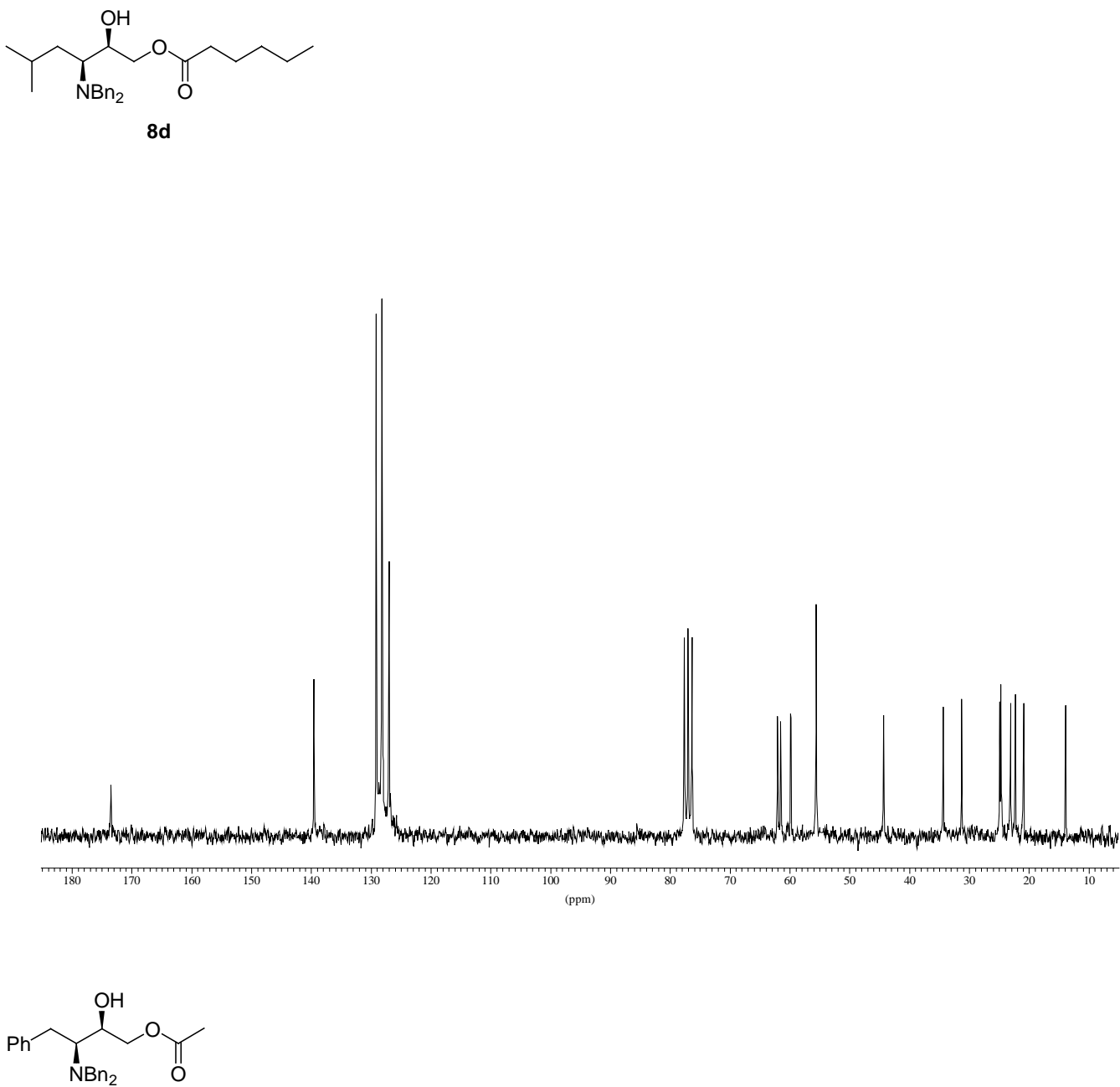

8 e

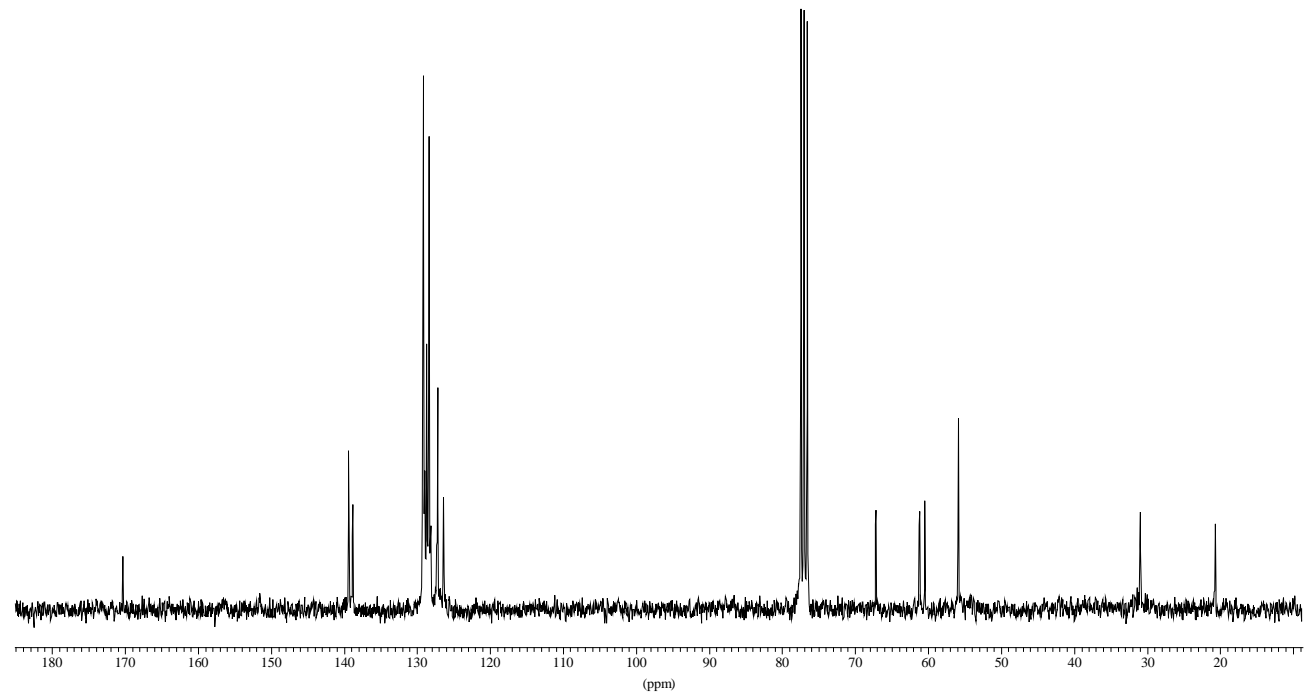



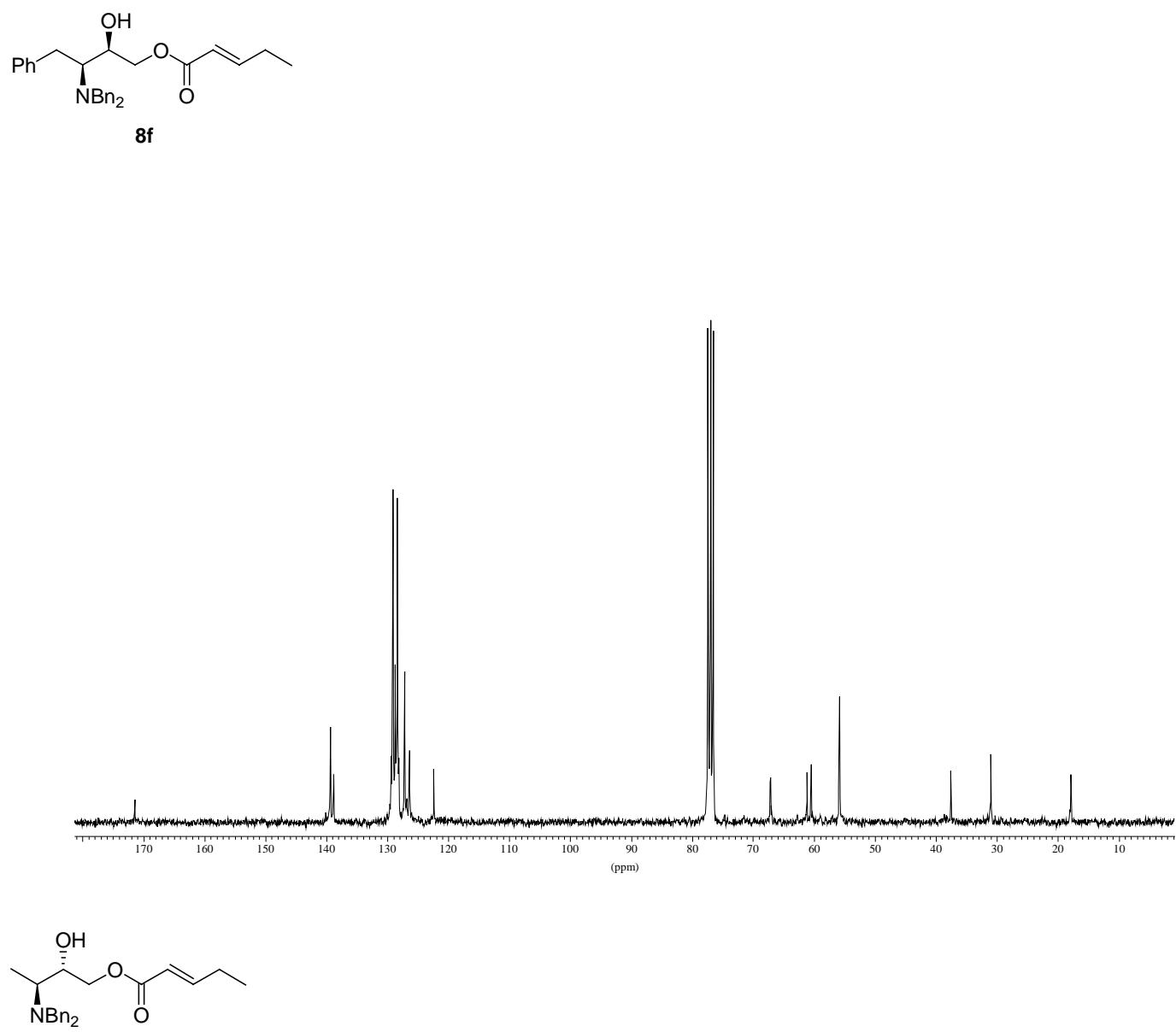

$9 a$

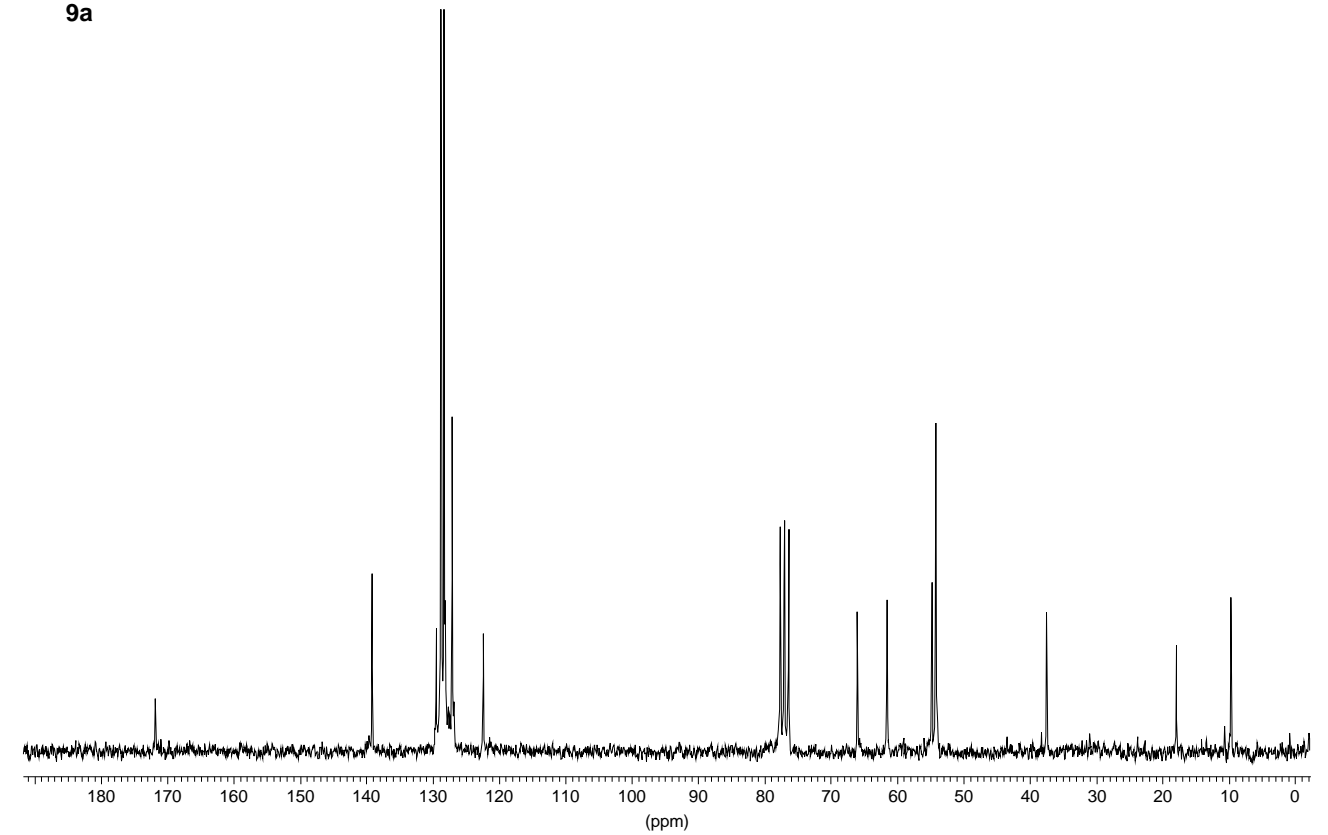



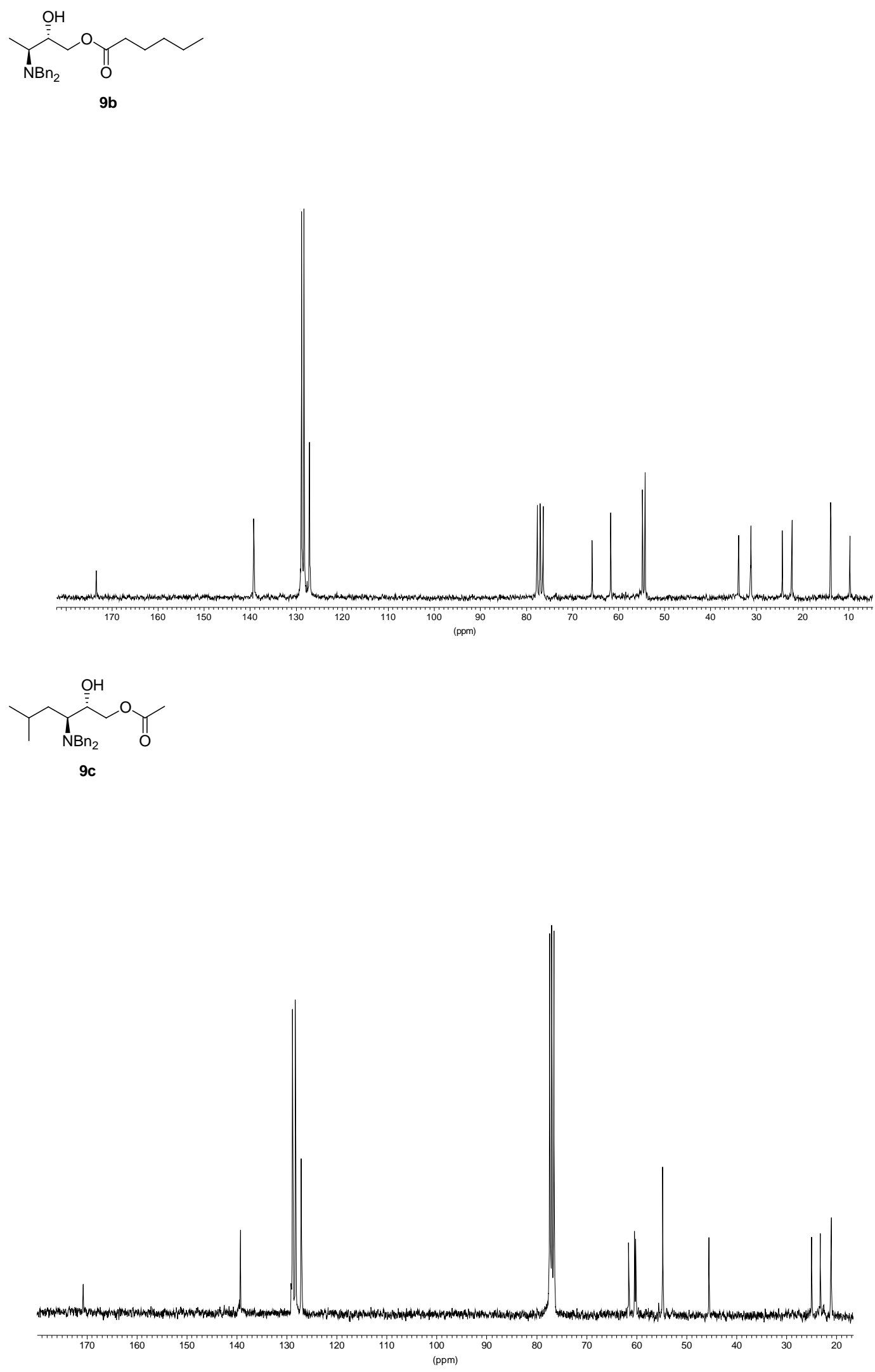

$8 \mathrm{~S}$ 

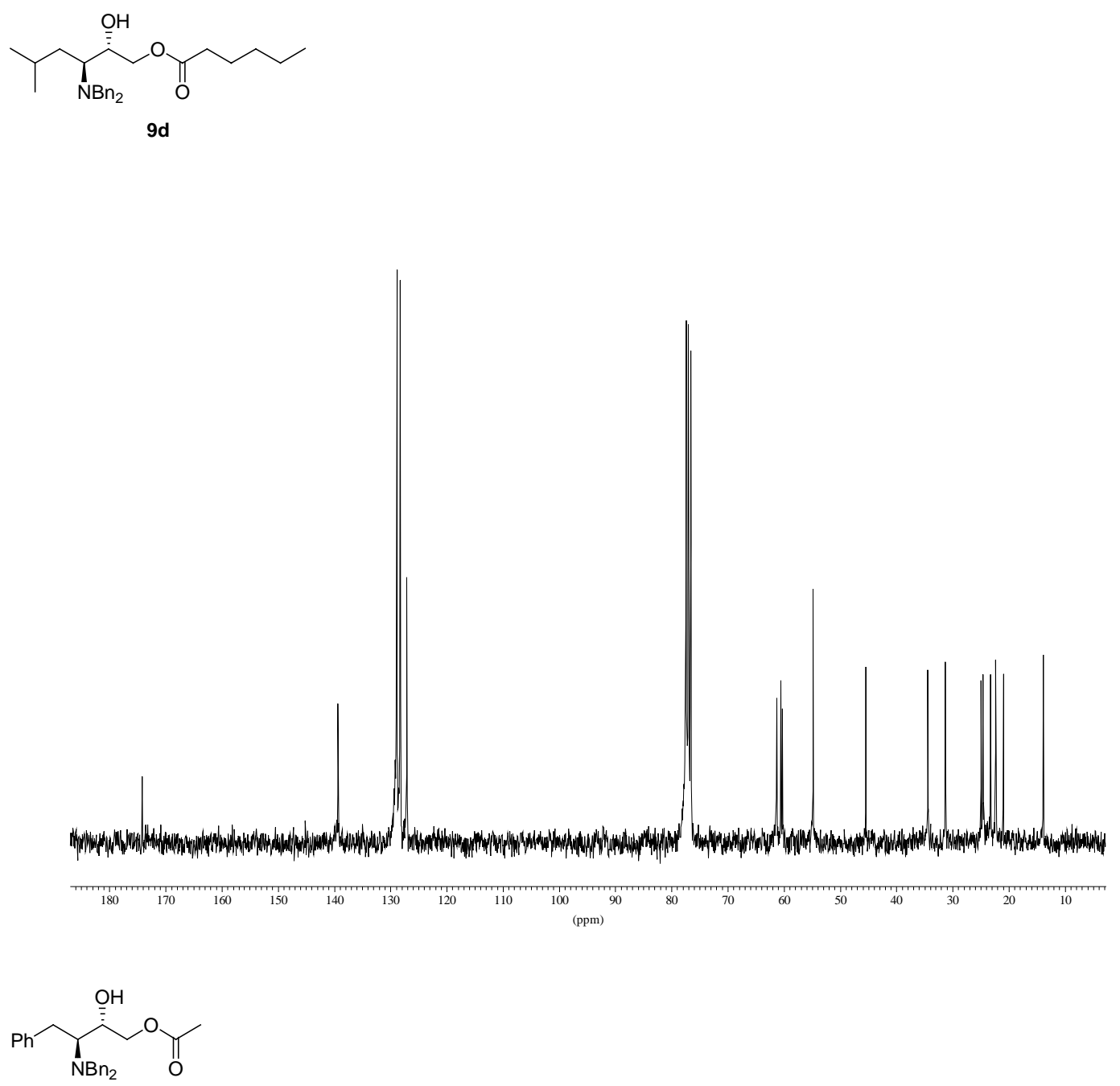

9 e

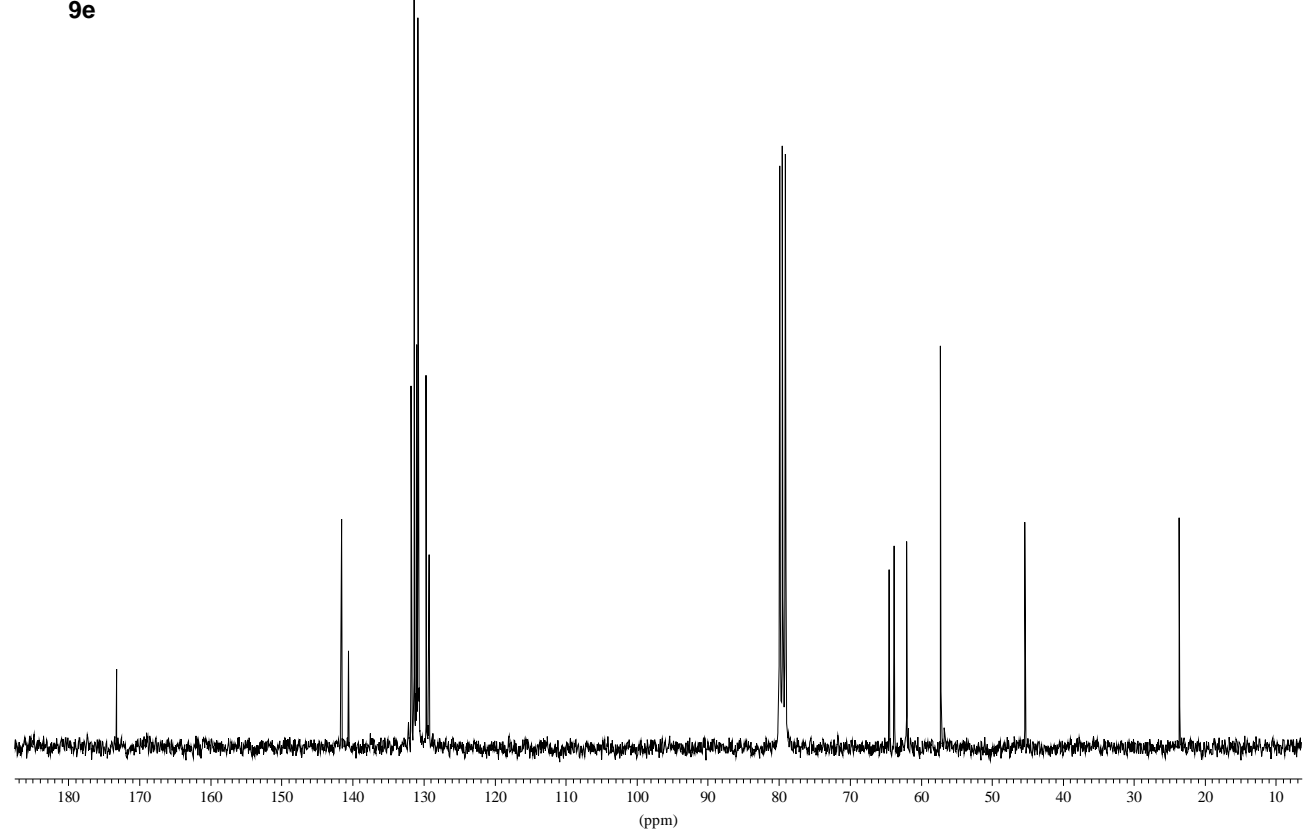



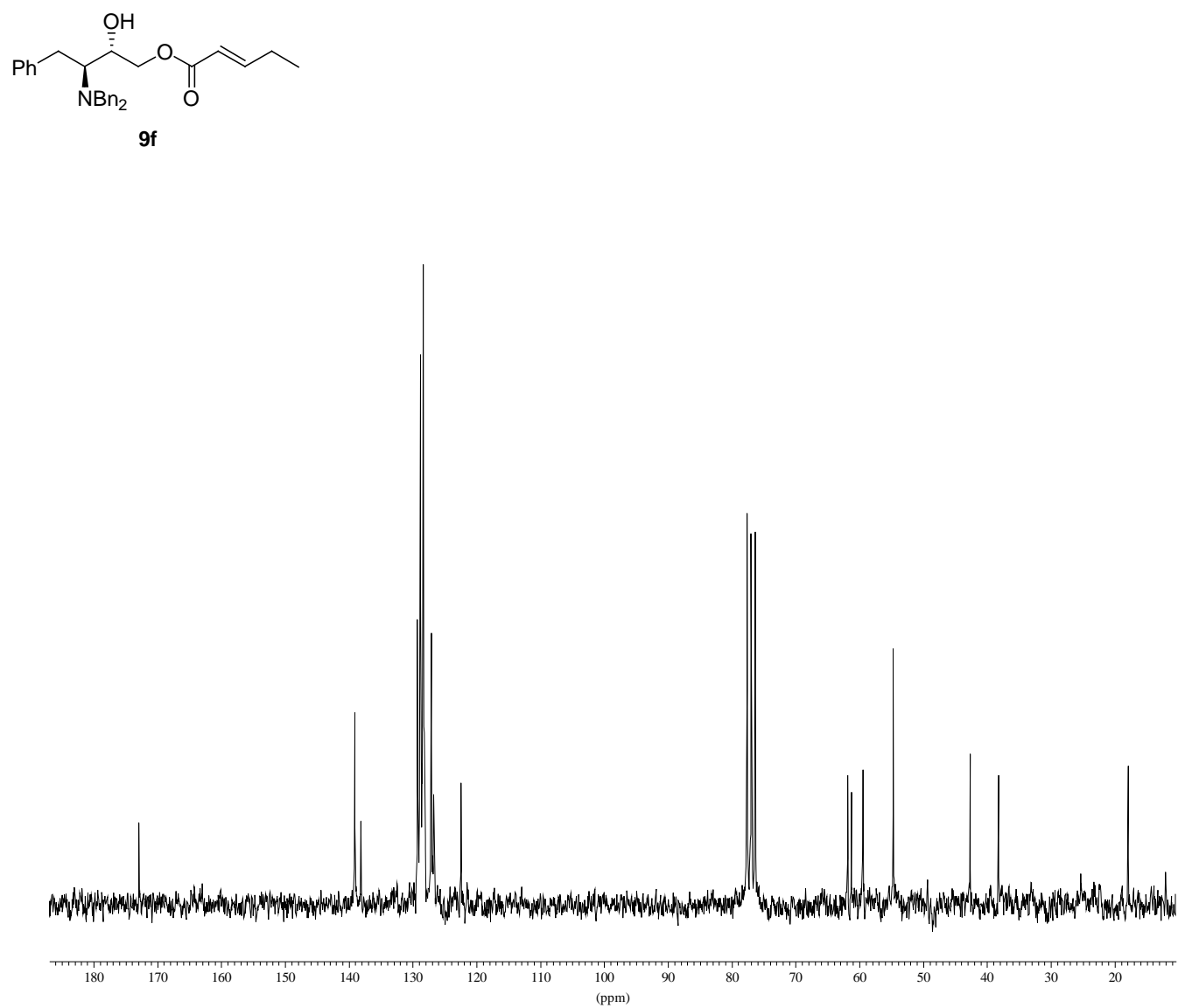